Oliveira F. y cols.

Rev. Chil. Pedlatr. 64 (5); 344-352, 1993

\title{
Dislipidemias en la infancia
}

\author{
Fernanda L.C. Oliveira ${ }^{1}$; Luis Paul F.G. De la Torre ${ }^{2}$; \\ María Paula Nolasco; Mauro Fisberg ${ }^{3}$
}

\section{Hyperlipemia in children}

\begin{abstract}
Non discriminale screenig of high blood cholesterol levels in children seems not to be justified, except in ihose coming from fomilies with high frequency of members affecled by hyperlipidemia, arterial hypertension, obesity, diobetes mellitus ond early onset coronary heart disease. Amang adull as well as children abese people with high risk foclors for alherosclerosis and coronary hearr diseose, changes in alimentary uses to diels with lower cholesterol and soturated fals logether with higher unsalurated fals contents may be beneficial, ever though there is not enough ovotable intormolion on the effecis of such a nutritional intervention on growth and development of the younger. Formacological iteatment, mainly with drugs that block bile acid reabsortion might be indicaled in same cases that do nol respond apropriately lo dis theropy alone.
\end{abstract}

[Key words: hypertipemia, cholesterol, anticholesteremic agents.]

Desde hace más de 30 años, los diferentes autores concuerdan en que la prevención de la arterioescletosis coronaria se debe iniciar desde la infancia ${ }^{1}$. La prevención precoz de la arterioesclerosis fue preconizada en 1985 por la Academia Americana de Pediatría. Kwiterovick ${ }^{2}$, fortaleció y sustentó la hipótesis del papel que ejerce el colesterol en la fisiopatología de las enfermedades cardiovasculares y de su importancia en el diagnóstico y tratamiento precoz.

Los avances en los estudios sobre el metabolismo lipídico pernitieron conocer las funciones de los lípidos, especialmente en el plasma, así como factores genéticos y ambientales que afectan su concentración. Los principales lípidos del plasma son colesterol, ésteres de colesterol, fosfolípidos y triglicéridos.

El colesterol tiene 27 átomos de carbono dispuestos alrededor de un núcleo esterol de cuatro anillos y un apéndice. Puede ser sintetizado por la célula animal a partir de la acetil CoA, actuando como catalizador la enzima hidroximetil-

1. Magister en Pediatría. Escola Paulista de Medicina.

2. Doctor en Pediatria. Escola Paulista de Medicina.

3. Doctor, Profesor Adjunto y Jefe de la Disciplina de Nutrición y Metabolismo, Departamento de Pediatría, Escola Paulista de Medicina. glutaril coenzima A reductasa (HMG CoA). Es un importante componente de las membranas celulares, además algunas células de mamíferos lo utilizan para producir ácidos biliares y hormonas esteroideas. El exceso de colesterol es almacenado en el interior de la célula, en forma esterificada. Cerca de dos tercios son sintetizados en el organismo (endógeno) y apenas un tercio proviene de la dieta (exógeno). El colesterol esterificado es un ácido graso que se encuentra ligado a un éster a través del carbono 3.

Los ácidos grasos son cadenas largas de átomos de carbono ligadas a átomos de hidrógeno. La nomenclatura de los ácidos grasos está indicada por la numeración de la cadena de carbonos a partir del extremo carboxilo (ácido) o por el otro extremo, que contiene el último átomo de carbono de forma insanrada omega. Los ácidos grasos son constituyentes esenciales de los triglicéridos y fosfolípidos, los cuales son hidrolizados y pueden ser oxidados por las células, para la producción de energía.

Los triglicéridos son lipidos que poseen una cadena de tres carbonos (glicerol), ligada a tres ácidos grasos por una unión éster. Los trigliceridos son sintetizados en las células del intestino, higado y tejido adiposo, y constituyen las fuentes dietéticas de grasa. 
En la circulación sanguínea los lípidos son transportados por lipoproteínas, a las que se clasifica en quilomicrones, lipoproteinas de muy baja densidad (VLDL), lipoproteínas de baja densidad (LDL), lipoproteínas de densidad intermedia (IDL) y lipoproteínas de alta densidad (HDL). Estas partículas son complejos lipídicos de macromoleculas, principalmente de colesterol esterificado, colesterol libre, triglicéridos, fosfolípidos y una familia de proteínas conocidas como apoproteinas (apo). Las principales apoproteínas son apo AI, apo I, apo IV, apo B-48, apo B-100, apo CII, apo C III y apo E.

\section{Metabolismo de las lipoproteinas}

Quilomicrones. Los quilomierones son secretados por las células de la mucosa intestinal. despuếs de la absorción de una dieta que contenga colesterol y triglicéridos. La apo B-48 constituye un componente esencial del quilomicrón, ya que lo torna viable para su secreción. Estas partículas llegan a la circulación sanguínea a través del sistema linfático mesentérico y del ducto torácico, donde sufren la acción hidrolítica de las lipasas, especialmente la lipasa lipoproteica (LPL), que necesita un cofactor para activarse, la apo CII(3). La apo CIII inhibiría la acción de la LPL(4). El quilomicrón remanente se mantiene rico en colesterol esterificado y en apo $E$, que es útil al hígado en la elaboración de colesterol endógeno. La apo CIII tendría un papel opositor a la apo $E^{5}$.

$V L D L$. Las fracciones de VLDL (pre-beta) son secretadas en los hepatocitos, sintetizadas a partir de pequefias moléculas de carbono y ácidos grasos libres que habían sido reutilizados por los tejidos periféricos. Los triglicéridos y colesterol provenientes de la dieta también pueden formar parte de la fracción interna de VLDL ${ }^{6}$. La apo B-ion tendría la capacidad de reunir y secretar $\mathrm{VLDL}^{\text {? }}$, permaneciendo una relación de una apo B-100 para cada partícula de VLDL, que sería convertida en IDL o LDL. En la circulación sanguínea la VLDL sufre la acción de la lipasa lipoproteica, hidrolizando triglicéridos hacia los tejidos periféricos. La VLDL remanente es transportada desde el plasma hasta el hígado por la apo E. Para la transformación de VLDL en IDL, LDL y VLDL remanente se necesitaría, además de la actividad de lipasa lipoproteica y de apo E, otra enzima fundamental, conocida como lipasa triglicerol hepática (HTGL) ${ }^{g}$.

LDL. La función de LDL (beta) es transportar el colesterol hacia los tejidos periféricos, para ser incorporado por las membranas celulares y por las hormonas en las estructuras gonadales y renales. La LDL actúa en la célula por intermedio de la apo B-100 con receptores celulares apo B, apo E y LDL, provocando la entrada de la partícula y la captación del colesterol libre por parte del citoplasma ${ }^{9}$. En las células que reciben el colesterol libre ocurre supresión de la síntesis del colesterol endógeno y de receptores LDL. La partícula de LDL puede ser captada por la célula, por una vía independiente de los receptores de superficie de membrana.

$H D L$. La fracción HDL (alfa) es una lipoproteina compleja, que posee un papel regulador en el transporte reverso del colesterol libre de las membranas celulares y el colesterol esterificado proveniente del colesterol libre del plasma. Se necesita de la lecitina colesterol acetiluransferasa (LCAT) para regular la esterificación de colesterol libre con la HDL ${ }^{10,11}$. Los fosfolípidos de la HDL pueden ser resecretados con la HDL nativa o estar asociados con la HDL circulante después del catabolismo de los quilomicrones y VLDL. La HDL se encuentra en dos subfracciones: HDL 3, partícula pequeña presente en el plasma en concentraciones constantes, pobre en proteína y de baja densidad, y HDL 2, de mayor tamaño, concentraciones plasmáticas muy variables, rica en triglicéridos y de densidad muy baja ${ }^{12}$. Las apoproteinas apo AI, apo AII y algunas apo $\mathrm{E}$ son parte integrante de las partifculas de HDL nativas del plasma; mientras que la apo CII, apo CIII y el resto de la fracción de apo E solamente quedan asociadas a HDL después que estas apolipoproteínas son transferidas de los quilomicrones y VLDL catabolizados. La enzima colesterol-éster proteína transferasa parece ser responsable de la transformación del colesterol esterificado de la HDL en VLDL, IDL y LDL y además, regularía la transferencia de los triglicéridos de las lipoproteínas ${ }^{13}$.

\section{Concepto y clasificación}

La hiperlipidemia es una alteración del mctabolismo de los lípidos y proteínas que causa aumento de los triglicéridos totales, colesterol total 
$y$ de la fracción LDL colesterol (valores superiores al percentil 95), y reducción de la fracción HDL colesterol en el plasma, con respecto a la distribución normal de las respectivas concentraciones plasmálicas de una determinada población según la edad y sexo. Un estudio realizado por el "Study Data Book" $1980^{14}$ estableció valores plasmáticos de lípidos y lipoproteínas para niffos y adolescentes, que fueron adaptados por la "American Heart Association" $1986^{15}$ (tabla). Según las orientaciones de Kwiterovich", el límite para considerar elevada la concentración de colesterol en la sangre durante las dos primeras décadas de vida es $200 \mathrm{mg} / \mathrm{dl}$ (normal $=170$ a $199 \mathrm{mg} / \mathrm{dl}$ ). Los valores normales de LDL colesterol plasmático estarían entre 100 y $129 \mathrm{mg} / \mathrm{dl}$.
Durante la primera década de vida las concentraciones plasmáticas de HDL se considerarían disminuidas entre 35 y $40 \mathrm{mg} / \mathrm{dl}$ y en la segunda década entre 30 y $35 \mathrm{mg} / \mathrm{dl}$. Las concentraciones plasmáticas de triglicéridos, tanto en la primera como la segunda década de vida, eran superiores a 100 y $130 \mathrm{mg} / \mathrm{dl}$ respectivamente.

Belamarich \& Deckelbaum ${ }^{17}$ consideran anormales en la infancia, concentraciones de colesterol total y LDL colesterol superiores a 200 y $135 \mathrm{mg} / \mathrm{dl}$, respectivamente. Según estos autores el colesterol al nacer sería bajo (40 a 90 $\mathrm{mg} / \mathrm{dl}$ ), aumenta rápidamente durante las primeras semanas de vida, después lentamente, para permanecer constante alrededor de los 2 afios de vida. Al nacimiento los triglicéridos varían entre

Tabla

Concentración lipídica plasmática (mg/dl)

Sexo masculino

Edad (años)

Colesterol

Triglicéridos

\begin{tabular}{lccccrr}
\hline & p5 & p50 & p95 & p5 & p50 & p95 \\
\hline 0 a 4 & 114 & 155 & 203 & 29 & 56 & 99 \\
5 a 9 & 121 & 160 & 203 & 30 & 56 & 101 \\
10 a 12 & 119 & 158 & 202 & 32 & 66 & 125 \\
15 a 19 & 113 & 150 & 197 & 37 & 78 & 148
\end{tabular}

\begin{tabular}{|c|c|c|c|c|c|c|c|c|c|}
\hline \multirow[t]{2}{*}{ Edad (Mños) } & \multicolumn{3}{|c|}{ HDL } & \multicolumn{3}{|c|}{ LDL } & \multicolumn{3}{|c|}{ VLDL } \\
\hline & p5 & p50 & p95 & p5 & p50 & p95 & p5 & p50 & p95 \\
\hline $\begin{array}{c}5 \text { a } 9 \\
10 \text { a } 14 \\
15 \text { a } 19\end{array}$ & $\begin{array}{l}38 \\
37 \\
30\end{array}$ & $\begin{array}{l}56 \\
55 \\
46\end{array}$ & $\begin{array}{l}75 \\
74 \\
63\end{array}$ & $\begin{array}{l}63 \\
64 \\
62\end{array}$ & $\begin{array}{l}93 \\
97 \\
94\end{array}$ & $\begin{array}{l}129 \\
133 \\
130\end{array}$ & $\begin{array}{l}0 \\
1 \\
2\end{array}$ & $\begin{array}{r}8 \\
10 \\
13\end{array}$ & $\begin{array}{l}18 \\
22 \\
26\end{array}$ \\
\hline
\end{tabular}

Sexo femenino

Edad (años)

Colesteral

Triglicéridos

\begin{tabular}{lllllll}
\hline & $\mathbf{p 5}$ & $\mathbf{p 5 0}$ & $\mathbf{p 9 5}$ & $\mathbf{p 5}$ & $\mathbf{p 5 0}$ & $\mathbf{p 9 5}$ \\
\hline $\mathbf{0 a 4}$ & 112 & 156 & 200 & 34 & 64 & 112 \\
5 a 9 & 126 & 164 & 205 & 32 & 60 & 105 \\
10 a 12 & 124 & 160 & 201 & 37 & 75 & 131 \\
15 a 19 & 120 & 158 & 203 & 39 & 75 & 132
\end{tabular}

\begin{tabular}{|c|c|c|c|c|c|c|c|c|c|}
\hline \multirow[t]{2}{*}{ Edad (años) } & \multicolumn{3}{|c|}{ HDL } & \multicolumn{3}{|c|}{ LDL } & \multicolumn{3}{|c|}{ VLDL } \\
\hline & p5 & p50 & p95 & ps & p50 & p95 & p5 & p50 & p95 \\
\hline $\begin{array}{c}5 \text { a } 9 \\
10 \text { a } 14 \\
15 \text { a } 19\end{array}$ & $\begin{array}{l}36 \\
37 \\
35\end{array}$ & $\begin{array}{l}56 \\
52 \\
52\end{array}$ & $\begin{array}{l}73 \\
70 \\
74\end{array}$ & $\begin{array}{l}68 \\
68 \\
59\end{array}$ & $\begin{array}{r}100 \\
97 \\
96\end{array}$ & $\begin{array}{l}140 \\
136 \\
137\end{array}$ & $\begin{array}{l}1 \\
2 \\
2\end{array}$ & $\begin{array}{l}10 \\
11 \\
12\end{array}$ & $\begin{array}{l}24 \\
23 \\
24\end{array}$ \\
\hline
\end{tabular}


37 y $56 \mathrm{mg} / \mathrm{dl}$, para después de 24 horas de vida comenzar a aumentar, probablemente debido al cambio de la fuente calórica de la glucosa por los ácidos grasos, y más de $50 \%$ del colesterol sería transportado por $\mathrm{HDL}^{18}$.

Webber y cols ${ }^{19}$ observaron en un período de 12 años los niveles de lípidos séricos de 1586 niños, con edades entre 2 y 14 años, encontrando disminución del colesterol total y LDL en la pubertad, principalmente en el sexo masculino, para luego aumentar hasta los 26 años. El colesterol HDL disminuyó después de los 14 años de vida. Los valores de triglicéridos permanecieron constantes durante la primera década de vida en niffos (as) de raza blanca y después de los 12 aflos de edad se registró un aumento.

Las hiperlipidemias pueden ser clasificadas en primarias y secundarias, considerándose primaria una vez que se han descartado las principales causas secundarias.

\section{Hiperlipidemias secundarias}

Las hiperlipidemias secundarias más frccuentes durante el primer año de vida son las producidas por enfermedades por almacenamiento de glicógeno y la atresia biliar congénita. Al final de la infancia predominan las de causas metabólicas como el hipotiroidismo, diabeles mellitus y síndrome nefrótico. Las causas exógenas aparecen principalmente durante las dos primeras décadas de vida.

Las causas secundarias de hiperlipidemias agudas y transitorias son las quemaduras y la hepatitis. Entre las enfermedades de depósito que las producen destacan las de cistina, glicógeno, enfermedad de Gaucher, enfermedad juvenil de Tay-Sachs, enfermedad de Niemann-Pick, enfermedad de Tay-Sachs. Las causas endocrinas y metabólicas incluyen porfuria intermitente, diabetes mellitus, hipopituitarismo, hipotiroidismo, lipodistrofia y embarazo. También producen hiperlipidemia secundaria causas exógenas como ingestión de alcohol, anticonceptivos, esicroides anabólicos, corticoides (prednisona), anticonvulsivantes, ciclosporina, ácido 13-cis-retinoico. Otra causa son las enfermedades hepáticas como la colestasis benigna intrahepática, atresia biliar congénita. También afecciones renales, entre ellas la insuficiencia renal crónica, síndrome hemolítico-urémico, síndrome nefrótico y otras más difíciles de clasificar, como anorexia nerviosa, hipercalcemia idiopática, síndrome de Kltnefelter, síndrome Hutchinson-Gilford (progeria), lupus critematoso sistémico, síndrome de Werner.

\section{Hiperlipidemias primarias}

Las hiperlipidemias primarias, fucron clasificadas por Fredrickson \& Levy ${ }^{20}$ según el fenotipo de la fracción lipídica que se encuentre aumentada en las formas que se identifican a continuación.

El Tipo I se caracteriza por triglicéridos elevados (TG > $1000 \mathrm{mg} / \mathrm{dl}$ ); hiperquilomicronemia muy infrecuente; deficiencia de lipoproteína lipasa o de apoproteína CII, herencia autosómica recesiva, con clínica abundante de intensa lipidemia (xantomatosis eruptiva, hepatoesplenomegalia, pancreatitis y retina lipémica).

En cl Tipo 11 el colcsterol total está clcvado entre 300 a $800 \mathrm{mg} / \mathrm{dl}$; LDL > $130 \mathrm{mg} / \mathrm{dl}$. En el subtipo Ila solamente hay aumento del colesterol y de la fracción LDL colesterol. En el subtipo IIb hay aumento del colesterol, la fracción LDL y los triglicéridos. Son ejemplos de éstas la hipercolesterolemia familiar y la combinada. En la hipercolesterolemia familiar (IIa o IV) el colestcrol está elevado (en homocigotos colesterol total 300 a $500 \mathrm{mg} / \mathrm{dl}$ y on heterocigotos colesterol total 400 a $800 \mathrm{mg} / \mathrm{dl}$ ), la incidencia es 2:1 000; ausencia, deficiencia a disfunción de los receptores LDL; enfermedad autosómica dominante, puede ser asintomática con xantomas plantares y tendinosos en los adolescentes o asociada a enfermedades vasculares en la infancia. En la hipercolesterolemia familiar combinada (IIb), hay aumento del colesterol (250 a $500 \mathrm{mg} / \mathrm{dl}$ ) y de los triglicéridos ( 250 a $750 \mathrm{mg} / \mathrm{dl}$ ), la incidencia cs 3 a 5:1 000, existe alteración de los receptores LDL y una apoproteina anómala en las VLDL, la enfermedad es autosómica dominante y asintomática hasta que aparece la enfermedad vascular.

En el Tipo III los triglicéridos y el colesterol total están elevados de 200 a $500 \mathrm{mg} / \mathrm{dl}$. La disbetalipoproteinemia familiar es ejemplo de este grupo, tiene una incidencia de alteración genética de 10:1000, con hiperlipidemia $<1: 1000$, apoproteina EIl anormal en LDL y función alterada de VLDL y IDL; es una enfermedad autosómica recesiva o poligénica. Asintomática hasta que 
aparece la enfermedad vascular. Se acompaña de xantomas palmares o eruptivos.

En el Tipo IV los triglicéridos se encuentran clevados a 250 o $750 \mathrm{mg} / \mathrm{dl}$, en ausencia de ayuno prolongado. La hipertrigliceridemia tiene una incidencia de 2:1 000, función anormal de VLDL, herencia autosómica dominante, es asintomática y con alto riesgo de enfermedad vascular.

El Tipo V corresponde a pacientes con triglicéridos y colesterol total elevados e hiperquilomicronemia. La hipertrigliceridemia con hiperquilomicronemia muestra aumento de triglicéridos (250 a $500 \mathrm{mg} / \mathrm{dl}$ ) y colesterol (250 a 500 $\mathrm{mg} / \mathrm{dl}$ ), muy infrecuente, deficiencia de apoproteĭna CII y de otro mecanismo todavía no definido, enfermedad autosómica dominante, puede presentar xantomas eruptivos, pancreatitis y ateroesclerosis.

La clasificación de Fredrickson \& Levy no incluye algunos tipos, descritos, de hiperlipidemia por disminución de HDL colesterol, que poseen características claramente definidas como la hiperlipidemia asociada con hipertrigliceridemia (HDL 20 a $35 \mathrm{mg} /$ dl y triglicéridos > 250 $\mathrm{mg} / \mathrm{di}$, función anormal de la HDL, asintonática y que puede llevar a un alto riesgo de enfermedad vascular); la hipoalfalipoproteinemia prímaria (HDL 5 a $35 \mathrm{mg} / \mathrm{dl}$ y triglicéridos $<250 \mathrm{mg} / \mathrm{dl}$, disturbio de la función de HDL, autosómica recesiva, puede ser asintomálica y frecuentemente asociada a enfermedades vasculares) y la hiperapobetalipoproteinemia.

Por la imposibilidad de incluir todos los tipos de hiperlipidemia en la clasificación antes mencionada, se optó por una clasificación metabólica de las dislipoproteinemias, que exige disponer de gran tecnología, provocando alto coste en los exámenes necesarios para su diagnóstico y las ordena en trastomos del metabolismo de LDL, de las liproproteínas ricas en triglicéridos y deficiencia de LDL.

1: Trastornos del metabolismo de LDL, incluyen los 1.a. por dismínución de la remoción de LDL (hipercolesterolemia familiar, defecto familiar de apo B-100), 1.b aumento de la producción de la LDL (hiperlipidemia familiar combinada, hiperapobetalipoproteinemia) y 1.c otros (hipercolesterolemia poligénica).

2: Trastornos de las lipoproteinas ricas en triglicéridos por 2.a disminución de la remoción (deficiencia de lipasa lipoproteica, deficiencia de lipoproteína CIL), 2.b producción anormal de
VLDL (hipertrigliceridemia familiar), 2.e disminución de remoción y aumento de producción (dislipoproteinemia, disbetalipoproteinemia) y 2.d otras (deficiencia de lipasa del triglicérido hepático, de proteína de transferencia del ester colesterol, elevación de los niveles de lipasa lipoproteica).

3: Deficiencia de HDL, que incluye 3.a aumen producción de HDL.

\section{Hipercolesterolemia familiar}

En la población de origen europeo la incidencia de hipercolesterolemia familiar es $0,2 \%$. Según Rey y cols ${ }^{21}$, la afección corresponde aproximadamente a $5 \%$ de las hipercolesterolemias. Es una enfermedad autosómica dominante. La incidencia de la forma heterocigota es aproximadamente $1: 250$ a 500 niños, mientras la homocigote no supera 1:1 000 000. Esta enfermedad puede ser causada por un defecto genético en el receptor celular de LDL, que provoca una alteración del catabolismo de las partículas LDL colesterol. El receptor LDL es una glicoproteína de membrana sintetizada como un precursor, que sería transportado del retículo endoplasmático hacia el complejo de Golgi, donde se forma la LDL madura. En los pacientes homocigotos se encontraron tres fenotipos celulares: negativos para el receptor LDL (sin afinidad de fijación al LDL), defecto en el receptor LDL (disminución de la capacidad de fijación al LDL) y defecto en la entrada de LDL (existe capacidad de fijar LDL, pero no para in troducirlo) $)^{22,23}$

Los niños heterocigotos parecen ser asintomáticos durante la primera década de vida. Se puede diagnosticar heterocigotos con hipercolesterolemia familiar utilizando sangre del cordón umbilical (L.DL > $41 \mathrm{mg} / \mathrm{dl}$ ), si alguno de los progenitores es portador de la enfermedad. A la edad de un año tendrán valores de colesterol total y LDL de 230 y $160 \mathrm{mg} / \mathrm{dl}$ respectivamente. Unicamente en 10 a $15 \%$ de los niños afectados se observa xantomas en los tendones durante la segunda década, principalmente en los tendones de Aquiles y extensores de las manos. En la adolescencia la tendinitis de Aquiles y las tenosinovitis pueden ser las primeras manifestaciones de la hipercolesterolemia familiar. Raramente los heterocigotos presentan "angina pectoris" en la primera década de vida. 


\section{Hipercolesterolemia familiar combinada}

En un disturbio del metabolismo de LDL que causa elevación de LDL apo B-100, 10 que desencadena gran número de partículas de LDL. La síntesis de apo B-100 y LDL se encuentra aumentada en el hígado, debido a la producción excesiva de VLDL. Clínicamente, puede presentarse como una enfermedad coronaria precoz. En relación a los estándares lipoproteicos puede ocurrir hipercolesterolemia (lla), hipertrigliceridemia (IV) o ambas (IIb).

\section{Hipertrigliceridemia familiar}

Es causada por una alteración autosómica dominante de penetración lenta. El fenotipo se expresa en 1:5 niños menores de 5 años que tengan un progenitor afectado por la enfermedad. Ellos presentan valores elevados de VLDL y triglicéridos con LDL prácticamente normal y HDL a veces disminuida. El hígado fabrica partículas VLDL de gran tamaño, con mayor cantidad de triglicéridos que la VLDL normal; así se explica que la producción de LDL sea adecuada.

\section{Disbetalipoproteinemia}

Ocurre por la presencia de VLDL anormal, con alteración en la composición química y movilidad electroforética beta. La causa parece ser el aumento de la cantidad de triglicéridos en las lipoproteínas, provocado por una depuración lenta. En condiciones normales la apo E se une al quilomicrón remanente y a los receptores LDL (BE) en la superficie hepática, causando la captación hepática de los quilomicrones remanentes y de VLDL. En este disturbio el aumento de los quilomicrones remanentes y la VLDL produce defectos que involucran un locus génico polimorfo, que especificaría la estructura de la apo E. Los pacientes son afectados al final de la segunda década, siendo raras las manifestaciones clínicas en la infancia y adolescencia. Se observan xantomas planos en las palmas de las manos y tuberosos en los codos, glúteos y rodillas, raramente xantomas tendinosos, como también ateromalosis precoz de las coronarias, carótidas, abdominales y femorales.

\section{Tratamiento de las hiperlipidemias}

Aspectos dietéticos. La prevención dietética implica educar a la población sobre la importancia de una alimentación balanceada, con menor contenido en grasa saturada, colesterol y sodio, con la finalidad de prevenir enfermedades cardiovasculares, arterioesclerosis e hipertensión.

Ел poblaciones de alto riesgo de enfermedades cardiovasculares, arterioesclerosis y obesidad es necesario incentivar la prevención de las hiperlipidemias, con vigilancia clínica del aumento de peso y rastreo del colesterol plasmático en la infancia, concomitantemente con estímulos para cambios del hábito alimentario de la familia (menor contenido de colesterol y grasa saturada). La restricción dietética no es aconscjable en los niños menores de dos años. La privación de algunos tipos de grasas puede causar daños tanto en la constitución de las membranas como en el desarrollo de la retina, o en el proceso de mielinización nerviosa, aunque una teoria sostiene que el organismo utiliza el colesterol endógeno durante el crecimiento y desarrollo normal, sin necesidad de adquirirlo por la alimentación ${ }^{24}$. Por orra parte no se conocen los probables efectos que tal restricción podría tener en la respuesta inflamatoria del sistema de inmunidad ${ }^{25}$.

En los casos de recién nacidos y lactantes homocigotos o heterocigotos con hipercolesterolemia familiar, la dietoterapia estaría indicada a pesar de todos los posibles riesgos de la medida. Para este efecto se usan fórmulas lácteas con menos colesterol, con grasa poliinsaturada, no excediendo de $10 \%$ y la estrecha colaboración de un profesional nutricionista para los cálculos de una dieta balanceada. En seguimientos de 10 años a nifos y lactantes que recibieron dietas restringidas no se demostraron alteraciones en el desarollo y crecimiento ${ }^{16}$. Sin embargo, al cabo de varios estudios realizados en animales y humanos para intentar establecer el efecto de una dieta restringida prolongada durante el primer año de vida, Deckelbaum ${ }^{25}$ sugiere actuar cautelosamente, comparando el riesgo de las alteraciones genéticas (hipercolesterolemia familiar) con las posibles influencias de este tipo de dieta sobre su crecimiento y desarrollo. La dieta de los niños hipercolesterolémicos deberá consistir en menores cantidades de grasa total, grasa saturada, colesterol y azúcares simples, con incremento de la ingestión de gtasas insaturadas y carbohidratos 
complejos. No es recomendable disminuir la ingestión total de proteínas.

Según la Asociación Americana (EUA) del Corazón $^{26}$, el tratamiento dietético para niños mayores de dos años consta de dos fases, según la distribución de los nutrientes con respecto a su proporción en el total de calorias:

Fase I: grasa total $30 \%$ (saturada $<10 \%$, poliinsaturada $10 \%$, monosaturada 10 a $15 \%$ ); colesterol $<300 \mathrm{mg} /$ día; carbohidratos 50 a $60 \%$; proteínas 10 a $20 \%$ de las calorias totales.

Fase Il: grasa total $30 \%$ (saturada $<7 \%$, poliinsaturada $10 \%$, monosaturada 10 a $15 \%$ ); colesterol < $200 \mathrm{mg} /$ día; carbohidratos 50 a $60 \%$; proteínas 10 a $20 \%$ de las calorias totales. El éxito del tratamicnto dietético dependerá de la educación del niño y su familia. La instrucción deberá ser orientada a of recer conocimientos para la substitución de hábitos, como grasas monosaturadas por poliinsaturadas, disminución del tamaño de las porciones, frecuencia y control de la ingestión diaria total ${ }^{24}$.

Los trastornos metabólicos responden a la dieta disminuyendo el nivel plasmático del colesterol total y LDL aproximadamente en 10 a $15 \%^{2}$. Otros autores concuerdan en que los valores plasmáticos de LDL colesterol pueden disminuir hasta $25 \%{ }^{15}$. Según el Instituto Nacional de Salud (EUA), el colesterol plasmático disminuyó aproximadamente $11,5 \%$ (variando de 2 a $18 \%$ ) en 10 homocigotos con hipercolesterolemia lamiliar $^{27}$. Los niños con intensa hipercolesterolemia necesitan de una dieta muy estricta, con aproximadamente $10 \mathrm{a} 15 \mathrm{~g}$ día de grasa, manteniendo una ingestión de ácido linoleico de $1 \%$ dc las calorías, así también como los que presentan hiperquilomicronemia necesitan de triglicéridos de cadena media ( $15 \%$ del total de calorias), que pueden ser absorbidos directamente a través de la vena porta ${ }^{28}$. En casos de hipercolesterolemia familiar on que no se consiguc disminuir la LDL colesterol a concentraciones menores al percentil 95, se puede usar una dieta pobre en grasa $(<25 \%)$, sabiendo los riesgos de esta medida ${ }^{17} . \mathrm{La}$ oferla diaria de calorías dependerá de la edad del paciente y de las necesidades para su crecimicnto (1 200 a 2300 calorías). En niños hi. pertrigliceridémicos y obesos (P/E $>120 \%)$, la disminución del peso ayudará en el tratamiento, utilizando el factor peso/estatura. Debe in- centivarse la práctica de ejercicios físicos, como largas caminatas por día. Estas medidas conjuntas auxilian a la terapéutica dietética.

Todavía se desconoce el efecto de una dieta rica en grasa poliinsaturada durante un tiempo prolongado, pero podría producir alteraciones como cálculos biliares y enfermedades malignas $^{16}$. Las dietas recomendadas son semejantes a las utilizadas normalmente por niños del Mediterráneo, como griegos, israelitas e italianos, sin presentar alteraciones del crecimiento y desarro$\mathrm{llo}^{24}$.

\section{Farmacología}

Cerca de $80 \%$ de los niños con hipercolesterolemia familiar necesitan tratamiento farmacológico, además de la dieta, para intentar controlar los niveles plasmáticos de colesterol y $\mathrm{LDL}$ colesterol ${ }^{16}$. Existen varias preguntas no resueltas sobre la utilización de la terapéutica farmacológica, pues no hay pruebas concretas de su capacidad para reducir el riesgo de enfermedad cardiovascular del adulto cuando se usan en la infancia; todavía se duda sobre el uso prolongado de medicamentos anticolesterol, debido a los cfectos colaterales que pueden provocar y tampoco están bien definidos los criterios para el inicio y tiempo de tratamiento ${ }^{17}$.

El tratamiento farmacológico está indicado cuando no se obtienen resultados satisfactorios con la dicta, por ejemplo al utilizar durante un año una diela con menor cantidad de grasa, la LDL sigue mayor que $160 \mathrm{mg} / \mathrm{dl}$, o cuando existe alto ricsgo de arterioesclerosis precoz en niños con hipercolesterolemia familiar ${ }^{17}$.

Las resinas secucstradoras de ácidos biliares son los medicamentos de elección para el tratamiento de las hiperlipidemias. La colestiramina disminuye la concentración plasmálica del colesterol, al unirse a los acidos biliares, causando eliminación fecal de los mismos y, en consecucncia, reducción de la reserva hepática de colesterol y aumento de la actividad del receptor de LDL en la superficie de los hepatocitos. Los efccios colaterales al empleo de resinas son sensación transitoria de plenitud gástrica, constipación, esteatorrea y posible mala absorción de vitaminas liposolubles (A,D,E,K). Los pacientes que utilicen este tipo de fármacos deben ser controlados ел sus concentraciones séricas de vita- 
minas liposolubles y del folato de eritrocitos, pruebas de función hepática y hemograma completo. Estos niffos tienen que recibir suplementos de polivitamínicos, hierro y ácido fólico.

Otras drogas que podrían ser utilizadas solamente en niffos de más de 5 a 7 años son el ácido nicotínico (disminuye el nivel plasmático del colesterol y aumenta la HDL), los fibralos, inhibidores de la HMG CoAR, d-tiroxina. Todas ellas no han sido aún satisfactoriamente probadas para su empleo en niños, donde pueden ocasionar múltiples efectos colaterales, molivo por el cual tienen que ser mejor estudiadas antes de indicarlas.

\section{Resumen}

La realización de exámenes de rastreo de colesterol en todos los niños no parece ser una realidad adecuada. En poblaciones de individuos adultos con obesidad, ricsgo elevado de ateroesclerosis y enfermedad coronaria, el cambio de hábito alimentario tanto en los niños como en los adultos, a una dieta con menor contenido de colesterol y grasas saturadas, parece una conducta adecuada, pero no se sabe con certeza si esta intervención dietética interferirá o no con cl crecimiento y desarrollo de los más jóvenes.

El diagnóstico de hiperlipidemia en la infancia parece estar directamente asociado con los antecedentes familiares de enfermedad coronaria precoz, obesidad, diabetes, hipertensión y trastomos hiperlipidémicos de los progenitores. La mejor forma de tratamiento consiste en la dietoterapia, basada en la ingestión de un menor contenido de grasas saturadas y colesterol, poco enriquecida de grasas poliinsaturada. La terapéutica farmacológica puede ser indicada en ciertos casos; las resinas secuestradoras de ácidos biliares son la mejor opción.

(Palabras clave: hiperlipemia, colesterol, agentes anticolesterémicos.)

\section{Referencias}

1. Reisman M: Arteriosclerosis and pediatrics. J Pediatr 1965: 66: 1-7.

2. Kwiterovich $J$ r $P O$ : Bile acid sequestrate resin therapy in children. In Fears $R$ (ed) Phamacology conirol of byperipidemia. Bancelona JR Prous Science Publishers. 1986; 55.
3. LaRosa JC, Levy RI \& Herbert $P N$ : A specific apoprotein activator for lipoprotein lipase. Biochem Biophys Res Commun 1970; 21: 942-952.

4. Ginsberg $I N$, Le NA \& Goldberg $I J$ : Apolipoprotein B metabolism in sunjects with deficiency of apolipoproteins apo CII and AI: evidence that apolipoprotein CIII inhibits catabolism of triglyceride-rich tipoproteins by lipoprocin lipase in vivo. J Clin Invesi 1986; 68 : 1287.1295 .

5. Quardfordt $S H_{i}$ Michalopoulos $G$ \& Schmirmer B: The effect of human $\mathrm{C}$ apolipoproteins in vitro bepatic metabolism of triglyceride emulsions in rat. J Biol Chem 1982; 257: 14642-14647.

6. Craig $W Y \&$ Cooper $A D$ : Effects of chylomicrons remnants and b-VLDL on the class and composition of newly secretcd lipoproteins by $\mathrm{HepG} 2$ cells. I Lipid Res 1988; 29: 299-308.

7. Mallory MJ, Kane JP, Hardman DA, Hamition RL \& Dalal KB: Normolriglyceridemic abetalipoproteinemia: absence of the B-100 apolipoprotein. J Clin Invest 1981 ; 67: $1441-1450$.

8. Goldberg IJ. Le NA, Ginsberg HN, Krauss RM \& Lindgren FT: Lipoprotein metabolism during acute inhibition of lipoprotein lipase in the cymomolgus monkey. J Clin Invest 1988; 81: 562-568.

9. Brown MS \& Goldste in $/ L$ : How LDL receptors influence cholesterol and atherosclerosis. Sci Am 1984; 251: 58-66.

10. Eisenberg $S$ : High density lipoprotein metabolism. J Lipid Res 1984; 25: 1017-1058.

11. Kostner GM, Knipping G, Groener JE, Zechner $R$ \& Dieplinger H: The role of LCAT and cholestheryl ester transfer proteins for the HDL and LDL struclure and metabolism. Ady Exp Med Biol 1987; 210: 79-86.

12. Miesenbock $G$ \& Pastch $J R$. Relationship of triglyceride and high-density lipoprotein metabolism. Atherosc Rev 1990: 21: 119-127.

13. Tall AR: Plasma lipid transfer proteins. J Lipid Res $1986 ; 27: 361-367$.

14. Lipid Research Clinic's Population Sikdy Data Book: The Prevalence Study Washington, D.C. U.S., Department Health and Human Services. Publication NIH. $1980 ; 80-1527$.

15. AHA Position Statement. Diagnosis and treatment of primary hyperlipidemia in childhood. Circulation. 1986: 74: $11 \mathrm{B2a}$.

16. Kwiterovich PO Jt.: Biochemical, clinical, epidemiologic, genelic and pathologic data relevant to the cholesterol hypothesis. Pediatrics. 1986; 78: 349-362.

17. Belamarich $P F$ \& Deckelboun $R J$ : Hypercholesterolemia in children: when to treat. Drug Therapy 1990; 41. 52 .

18. Nestel PJ: Nutrición en cl metabolismo lipídico y las hiperlipjdemias. En Nulrición Clínica en la Infancia. Testlé Nulrition, Vevey/Raven Press, New York: 1987: 195-215.

19. Webber LS, Srinivasan SR, Wattigney WA \& Bereson GS: Tracking of serum lipids and lipoproteins from childhood to adulthood. The Bogalusa Hearth Study. Am I Epidemiol 1991: 133: 884-899.

20. Fredrikson DS, Levy RI \& Laes RS: Fat transpon in lipoprotein: An inlegrated approach to mechanisns and disorders. New Engl I Med 1967: 276: 34-42. 
21. Rey J, Farriaux JP, Lenoir Ge cols: Les hyperlipoproteinemies al la prevention de láherome chez enfants. XXV Congres Française Languague of Pediatrician. Tolouse. France. Fourmier 1981: 2: 227-291.

22. Goldstein $M L$ \& Brown MS: Familial Hypercholesterolemia. In Scriver CR, Beaudet AL, Sly WS \& A] ed: The metabolic basis of inherited disease, ed 6, vol. 1 New York. McGraw-Hlill 1989; 1215.

23. Brown $M S$ \& Goldsie in $J$; A receptor mediated parhway for cholesterol homeostasis. Science 1986; 232: 34.

24. Deckelbawn $R J$ : Delecting and treating hyperlipemis in children. Medical Management Dynamics 1990, 8(1):6-12.

25. Deckelbown $R J$ : Long-term effects of diet in first year of life on cholesterol metabolism and atherosclerosis. In Nutrition needs of six to twelve month ol infant.
Heird WC (ed) Camation Nutrition Education Series. Vol 2 Carnation Co., Clendale/Raven Press, New York 1991; 297-302.

26. AHA Position Statemenl: Guidelines for Healthy American Adults. A Statement for Physicians and Health Professionals by the Nutrition Committee, Publ \# $\gamma_{1}$ 1003 Dallas, Texss, 1988.

27. Levy RI. Frederikson DS and Shulmon R: Dietary and drug treatment of primary hiperlipoproteinemia. Ann Iniem Med 1972: 77: 267-271.

28. Kwiterovich Jr $P O$. Diagnostico e tratamento da dislipoproteinemia familial em crianças e adolescentes. In Problemas atuais na Endocrinología Pediátrica e Adolescente. Clínicas pediátricas da Anérica do Norte 1990; 6: $1609-1645$. 